\title{
Video Self-Modelling to Reduce Aggressive Behaviours in Adolescents with High Functioning Autism
}

\author{
Sandra Kinsella ${ }^{1, a}$, Wendi Beamish ${ }^{1, b}$ and Helen Klieve ${ }^{1, c}$ \\ ${ }^{1}$ School of Education, Griffith University, 176 Messines Ridge Road, Mt Gravatt QLD 4122, Australia. \\ askins6@eq.edu.au, b'w.beamish@griffith.edu.au, ch.klieve@griffith.edu.au
}

Keywords: video self-modelling, iPad, autism, High Functioning Autism, ASD, aggressive behaviour

\begin{abstract}
Primarily this study investigated the use of Video Self-Modelling (VSM) as a behaviour intervention strategy to reduce the occurrence of aggressive behaviours in three adolescents with High Functioning Autism (HFA) within a school environment. A single-subject non-concurrent multiple baseline design was employed in the form of a baseline-intervention-withdrawal (A-B-C) across three participants. The intervention engaged each participant with a personalised iPad-presented movie allowing them to observe self-modelled, positive behaviour choices across situations where aggression had previously been the response. Findings yielded positive outcomes for all participants indicating that VSM is an effective strategy for reducing aggressive behaviours in adolescents. This small-scale study builds on the existing research into VSM and contributes to the limited number of studies currently available for behaviour intervention with adolescents with high functioning autism.
\end{abstract}

\section{Introduction}

Autism Spectrum Disorder (ASD) is a multifaceted social and communication disorder that impacts on the ability of a young person's ability to communicate and navigate complex social situations [1] and these difficulties are often associated with social isolation, educational disengagement and the presence of challenging behaviours. Researchers have made a number of connections between a diagnosis of ASD and the presence of aggressive behaviours with some stating that a diagnosis of ASD can forecast the presence of such behaviours [2]. For young people with ASD and those around them, aggressive behaviours can be unsafe, socially unacceptable and over time can significantly impact the future life goals of the individual $[3,4]$ yet there is limited research into how to manage aggressive behaviours in individuals with ASD, particularly during adolescence.

\section{High Functioning Autism and Aggression}

A number of studies suggest that aggression and the self-regulation of anger are problematic issues amongst individuals with HFA [ 5,6,7]. Yet the majority of the research into aggression and ASD has been limited to the frequency and characteristics of such behaviours in young children with low functioning autism. ASD is a spectrum of disorders and by nature impacts on each individual differently thus a range of personalised and needs-driven supports, services and intervention strategies are required [8].

Many young people with ASD express a preference for learning through games and engagement with computers over human interaction as they find it difficult to ignore information in the environment that is unrelated to the learning particularly if it involves social interaction. Video modelling (VM) and Video Self-Modelling (VSM) are two intervention strategies that capitalise on this preference.

VSM appears particularly well suited to young people with ASD as it appeals to the egocentricity of the person and their high level of interest ICTs. VSM has been successful across an ever increasing range of behaviours, disabilities and ages [9]. 


\section{Setting and Participants}

The study engaged three male students from a large regional secondary school who met the five predetermined criteria: (1) a formal diagnosis of ASD (2) aged between 11 and 17 years (3) a Full Scale Intelligence Quotient (FSIQ) score not within the impaired range 4) a documented history of aggressive behaviours within the school environment 5) a previously expressed willingness/desire to cease aggressive behaviours. At the commencement of the research participants were aged between 12 and 14 years of age and the target behaviour was defined in a manner that was observable, tangible and measureable as outlined in Table 1.

Table 1. Participant overview and working definition of target behaviours.

\begin{tabular}{|c|c|}
\hline Participant & Target Behaviour \\
\hline $\begin{array}{l}\text { Participant } 1(\mathrm{P} 1) \\
\text { Age: } 14 \text { years } \\
\text { FSIQ - Normal range }\end{array}$ & $\begin{array}{l}\text { When unhappy with a direction or the outcome of an event, P1 yells and swears at peers and/or staff, } \\
\text { moving towards them in a threatening manner. He calls the person names, swear, threaten harm and } \\
\text { wave his arms about. He paces towards, then away from, the person he sees as the source of his } \\
\text { frustration. This event typically last a few moments. }\end{array}$ \\
\hline $\begin{array}{l}\text { Participant } 2(\mathrm{P} 2) \\
\text { Age: } 12 \text { years } \\
\text { FSIQ - Borderline } \\
\text { range }\end{array}$ & $\begin{array}{l}\text { When redirected by an adult P2 yells or swears at the person. When redirected or asked to take time } \\
\text { out he refuses and continues to swear and posture in an aggressive manner toward staff member. He } \\
\text { pushes his chest out, jumps or skips on the spot and pushes his arms back. When directed to stop he } \\
\text { often begs for a second chance and promises to behave however when this is refused he will strike } \\
\text { out at the air elevating further. When a phone call is made requesting assistance he rushes towards } \\
\text { staff, pushing past them to hang up the phone. He often pushes adults to move them away from the } \\
\text { telephone. }\end{array}$ \\
\hline $\begin{array}{l}\text { Participant } 3(\mathrm{P} 3) \\
\text { Age: } 14 \text { years } \\
\text { FSIQ - Normal range }\end{array}$ & $\begin{array}{l}\text { When passed, spoken to or approached by a peer whom P3 perceives as an antagonist he becomes } \\
\text { verbally abusive towards that person. If this person is staff member he verbally abuses them while } \\
\text { walking away. If it is a peer he invites them to fight. This is a loud and highly aggressive response } \\
\text { coupled with a high level of posturing and at times pushing with his chest. Should the person be } \\
\text { non-responsive he continues the behaviour until they push him away, lash out or engage in verbal } \\
\text { sparring. Once they respond verbally P3 responds physically punching at the head without concern } \\
\text { for consequence to himself or others. }\end{array}$ \\
\hline
\end{tabular}

\section{Method}

The present study employed a single-subject non-concurrent multiple baseline design [10,11] within the overarching methodology of Single Case Design (SCD). The baseline phase (A) consisted of the collection of data pertaining to the frequency of the target behaviour with the purpose of analysing data trends in order to establish an overall view of frequency and influencing circumstances across the school day/week. The intervention phase (B) of the present study consisted of three segments (B1, B2, B3). B1 consisted of a collaborative review of aggressive behaviours with the participant and the researcher in conjunction with the planning and development of video of a mutually agreed replacement behaviour; B2 comprised the planned viewing of the video over a maximum of five, preferably consecutive, school days; and B3 included the withdrawal of planned viewings but allowed continued opportunity to view the video for a subsequent ten school days. The withdrawal phase of the intervention $(\mathrm{C})$ commenced two weeks after the final planned viewing for each participant, with data collection planned for a further 20 school days.

\section{Video Production}

A combination of feedforward and positive self-review modelling was utilised to create individualised movies that were between two and three minutes in length $[12,13]$. Videos were created in collaboration with each participant and edited by the researcher to create a positive visual representation of the desired behaviour choice. The participants assisted with the selection of music, sound and visual effects. Support was provided in identifying moments in the video where positive choices were demonstrated and this was reinforced through the addition of titles and sound effects. Data collection and analysis was an interconnected process of collection and examination across all 
phases of the present study and all data was graphed in simple line graphs and visually scrutinised every 10 days to identify and compare data patterns within and across phases.

\section{Results}

During the baseline phase (A) all participants showed a persistent and high presence of target behaviour; however, a consistent decreasing data trend can be observed for all participants during intervention (B) and withdrawal (C) phases (refer Fig. 1). While P1 and P2 presented with a significant elevation in their target behaviour during the first segment (B1) of the intervention phase this was followed by a significant drop in the rate of behaviour once the planned viewing of their personalised iPad-presented movies commenced. A different behaviour pattern can be observed for P3, whose targeted behaviour reduced once discussion commenced during the first segment (B1) of the intervention phase. During the remaining segments of the intervention phase all participants demonstrated a reduction in the rate of the target behaviour. Both P1 and P3 recorded a limited number of events during this phase of this study. While P2 displayed a reduction in the rate of behaviour he continued to exhibit a higher level of variability compared to the other participants. Across the withdrawal (C) phase of this study all participants recorded a decreasing trend in the rate of target behaviour with $\mathrm{P} 1$ and $\mathrm{P} 3$ recording very low rates of behaviour.
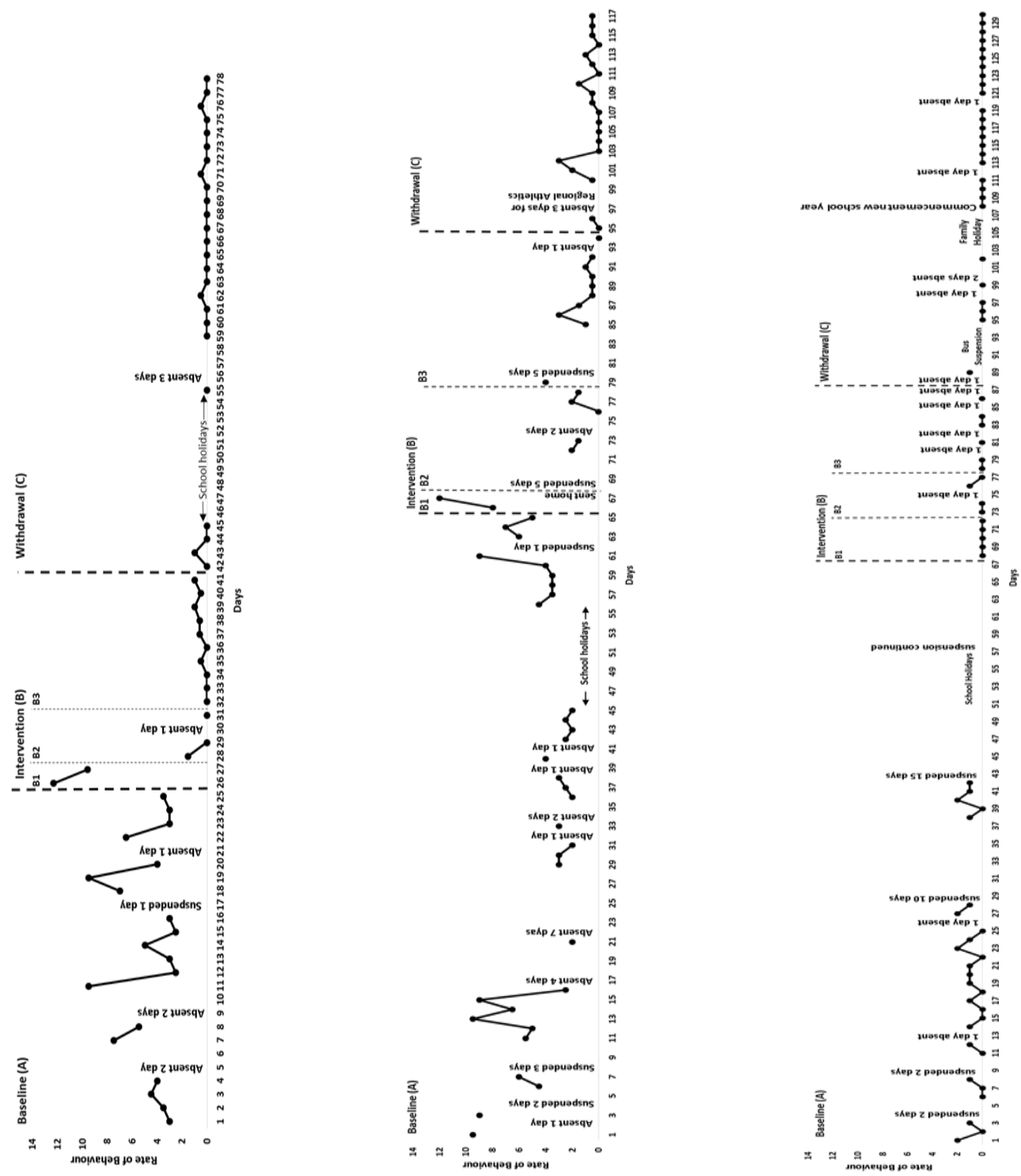

Fig. 1 A comparison of the rate of target behaviour across participants across all phases (A, B, C). 


\section{Conclusion}

Taken together these findings provide evidence supporting VSM as an effective intervention to reduce aggressive behaviours in adolescents with HFA. The use of VSM as a behaviour intervention offers a collection of benefits for teachers, other professionals and students with ASD. This study was implemented under natural conditions within a secondary school setting. Overall VSM proved to be a practical and easy to implement intervention strategy. A number of potential areas for future research have emerged through this research. It would be worthwhile replicating this study to further confirm the success of VSM as an intervention strategy to address complex and challenging behaviours in adolescents on the autism spectrum. The aspect of maintenance of interventions outcomes across time also needs further investigation.

\section{References}

[1] J. Brosnon, O. Healy, A review of behavioral interventions for the treatment of aggression in individuals with developmental disabilities, Research in Developmental Disabilities. 32 (2011) 437-446.

[2] K. McClintock, S Hall, C. Oliver, Risk markers associated with challenging behaviours in people with developmental disabilities: A meta-analytic study, Journal of Intellectual Disability Research. 47 (2003)405-416.

[3] J. Jang, D. Dixon, J. Tarbox, and D. Granpeesheh, Symptom severity and challenging behavior in children with ASD, Research in Autism Spectrum Disorders. 5 (2011) 1028-1032.

[4] M. Kaartinen, K. Puura, M. Helminen, et al. Reactive aggression among children with and without autism spectrum disorder, Journal of Autism and Developmental Disorders. 44 (2014) 2383-2391.

[5] S. Farrugia, J. Hudson, Anxiety in adolescents with Asperger syndrome: Negative thoughts, behavioral problems and life interference, Focus on Autism and Other Developmental Disabilities. 21 (2006) 25-35.

[6] S. Kanne, M. Mazurek, Aggression in children and adolescents with ASD: Prevalence and risk factors, Journal of Autism and Development Disorders. 41 (2011) 926-937.

[7] C.E. Pugliese, B.A. White, S.W. White and TH Ollendick, Social anxiety predicts aggression in children with ASD: Clinical comparisons with socially anxious and oppositional youth, Journal of Autism and Developmental Disorders. 43 (2012) 1205-1213.

[8] D.R. Hendricks, P. Wehman, Transition from school to adulthood for youth with autism spectrum disorders, Focus on Autism and Other Developmental Disabilities. 24 (2009) 77-88.

[9] T. Buggey, L. Ogle, Video self-modelling. Psychology in the Schools, 49 (2012) 52-70.

[10] T. Christ, Experimental control and threats to internal validity of concurrent and non-concurrent multiple baseline designs, Psychology in the Schools. 44 (2007) 451-459.

[11]B.D. Winn, C.H. Skinner, J.D. Allin, and J.A. Hawkins, Practicing schol consultants can empirically validate intervention: A description and demonstration of the non-concurrent multiple baseline design, Journal of Applied Scholl Psychology. 20 (2004) 109-128.

[12] T. Buggey, Seeing is believing: Video self-modeling for people with autism and other developmental disabilities, Woodbine House, Bethesda, MD, 2009.

[13] P.W. Dowrick, Self-modeling, in: P. W. Dowrick, J. Biggs (Eds.), Using video: Psychological and social applications, New York, Wiley, 1983. 